\title{
The structure of liquid metals probed by XAS
}

\author{
Adriano Filipponi ${ }^{1, *}$, Andrea Di Cicco $^{2}$, Fabio Iesari ${ }^{2}$, and Angela Trapananti ${ }^{3}$ \\ ${ }^{1}$ Dipartimento di Scienze Fisiche e Chimiche, Università dell' Aquila, I-67100 L'Aquila, Italy \\ ${ }^{2}$ Scuola di Scienze e Tecnologie, Sezione di Fisica, Camerino I-62032, Macerata, Italy \\ ${ }^{3}$ CNR-Istituto Officina dei Materiali-Perugia, I-06123 Perugia, Italy
}

\begin{abstract}
X-ray absorption spectroscopy (XAS) is a powerful technique to investigate the short-range order around selected atomic species in condensed matter. The theoretical framework and previous applications to undercooled elemental liquid metals are briefly reviewed. Specific results on undercooled liquid $\mathrm{Ni}$ obtained using a peak fitting approach validated on the spectra of solid $\mathrm{Ni}$ are presented. This method provides a clear evidence that a signature from close packed triangular configurations of nearest neighbors survives in the liquid state and is clearly detectable below $k \approx 5 \AA^{-1}$, stimulating the improvement of data-analysis methods that account properly for the ensemble average, such as Reverse Monte Carlo.
\end{abstract}

\section{Introduction}

The development of methods that provide access to the undercooled liquid state, combined with the availability of improved x-ray and neutron radiation sources, has stimulated a novel interest in experimental investigations of liquid metals. Containerless materials processing techniques such as electromagnetic levitation [1] or aerodynamic levitation [2] were exploited to perform x-ray or neutron diffraction [3-5] experiments in a clean environment reaching a deep undercooling in metallic melts.

X-ray absorption spectroscopy (XAS) $[6,7]$ is a powerful technique to probe the shortrange structure around selected atomic species in condensed matter including the liquid state $[8,9]$. The typical bulk sample thickness required to optimize transmission experiments for transition metal K-edges is of the order of 10-20 $\mu \mathrm{m}$ therefore suitable samples can be prepared as a dispersion of micrometric sized grains embedded in a supporting matrix [10]. Even if this environment is not as clean as in the case of levitation techniques, a deep undercooling can be usually achieved because of the small droplet size, in particular when the supporting framework does not act as a strong heterogeneous nucleant. Our contribution to the field includes a number of structural investigations on undercooled liquid elemental metals with high melting points such as $\mathrm{Pd}$ [11], $\mathrm{Rh}$ [12], $\mathrm{Cu}$ [13], Ni [14] at high temperature and low melting point metals such as Sn [15] and Ga [16] at high pressure.

The undercooling capabilities can be assessed using x-ray absorption temperature scans [17] and these latter measurements exploited to determine the specific crystal nucleation rate [18]. Recent Ni data [14] were further investigated to assess the possible occurrence of homogeneous nucleation [19] with the support of kinetic Monte Carlo simulations [20].

\footnotetext{
*e-mail: adriano.filipponi@aquila.infn.it
} 
The focus of this paper is on the nature of the short-range structural information in x-ray absorption spectra measured in the liquid phase and in particular on the assessment of the presence and intensity of signals associated with triplet correlations. The presence of a signature from close packed equilateral triangular configurations has been previously emphasized in the $\mathrm{Cu}$ case [13]. In this paper Ni K-edge XAS spectra at $T=1493 \mathrm{~K}$ in the solid and undercooled liquid phases [14] are analyzed with a peak fitting approach.

\section{Theoretical framework}

XAS has been extensively reviewed $[6,7]$. The photo-absorption cross section is dominated by one-electron dipole allowed transitions in which a core state electron ( $1 s$ for K-edges) is excited to unoccupied continuum states. The photon energy $\hbar \omega$ determines the photo-electron wavevector modulus $k=\frac{1}{\hbar} \sqrt{2 m\left(\hbar \omega-E_{0}\right)}$ where $E_{0}$ is the continuum threshold energy. The process occurs in the fs timescale, therefore the measured cross section probes an ensemble average over the instantaneous atomic positions and cannot access dynamical information. The final state photo-electron wavefunction is a continuum state at $10-1000 \mathrm{eV}$ above $E_{0}$ in a space containing the photoabsorber and the surrounding atoms. Current approximations for the cross-section calculation adopt an effective potential for the photoelectron which includes Coulomb and exchange-and-correlation terms from the surrounding charge density. The effect of the surrounding atoms is taken into account within a multiple scattering formalism. In the most effective approaches the usage of a muffin-tin approximation for the potential allows to separate the effects of the atomic species, represented by spherically symmetric potentials and related phase shifts, that are independent from the actual atomic positions, from the geometrical information. The origin of the oscillations in the cross-section, usually referred to as x-ray absorption fine structure (XAFS), is associated with interference effect from scattered waves from the surrounding atoms and therefore contains a valuable information on the short-range structure. Moreover, the strong non-linear relation between geometry and crosssection makes the dimensionless oscillating term $\chi(k)=\sigma / \sigma_{0}-1$ sensitive to contributions beyond the pair correlations ( $\sigma_{0}$ represents the atomic cross-section). It is possible to write a useful expansion of the signal in terms of irreducible pair, triplet, and higher order terms:

$$
\chi(k)=\sum_{i} \gamma^{(2)}(0, i)+\sum_{(i, j)} \gamma^{(3)}(0, i, j)+\sum_{(i, j, l)} \gamma^{(4)}(0, i, j, l)+\cdots
$$

Each of the $\gamma^{(n)}(0, i, \ldots)$ signal is an oscillating function with a leading phase proportional to $k R_{p} R_{p}$ being the length of the shortest (or dominant) path involving all atoms in the configuration. This includes the dominant pair contributions with $R_{p}=2 R$, where $R$ is the corresponding shell distance. The actual expressions for the $\gamma^{(n)}(0, i, \ldots)$ signals are not a simple functions and involve series expansions over angular momenta and related phase shifts for the atoms involved [21, 22]. In the case of disordered systems the sums in Eq. (1) can be evaluated using appropriate integrals over the pair, triplet, ..., atomic distributions [8].

\section{Validity assessment of the underlying approximations}

The simplest strategy to verify the accuracy of the present computational scheme is to attempt to model spectra of known structures in which the effect of the configurational average does not represent an unknown. Useful comparisons can be performed with the spectra of molecules in the gas phase [23] or of simple crystalline structures [22]. For this specific investigation is it clearly important to verify how the theory can account for the spectrum of crystalline f.c.c. Ni. The comparison is usually performed on the signal, conveniently plotted 
as $k^{n} \chi(k)$ where $n$ is a suitable integer power chosen to magnify the higher $k$ range of the spectra (for graphical reasons). The corresponding Fourier transform magnitude is evaluated with an $\exp (i 2 k R)$ exponential in such a way that the conjugated variable $R$ corresponds roughly to the physical distance (apart from a phase shift contribution which shifts the peaks to lower $R$ distances by about $0.4 \AA$ ).

The XAFS signals in an f.c.c. structure are dominated by the first shell single scattering contribution with a low frequency in $k$ space and peaking around the first shell distance in $R$ space. The intermediate range order instead contributes with a number of pair and triplet signals associated with triangular arrangements of first neighbor bonds at $60^{\circ}, 90^{\circ}, 120^{\circ}$, and $180^{\circ}$. The longer distances in the latter three triangular configurations correspond to second, third and fourth neighbors shells. All the above signals can be calculated for a reference configuration and the effect of atomic vibrations accounted for by a suitable complex damping term. The fit of the solid Ni $k \chi(k)$ spectrum, measured at $T=1493 \mathrm{~K}$ [14], including all pair
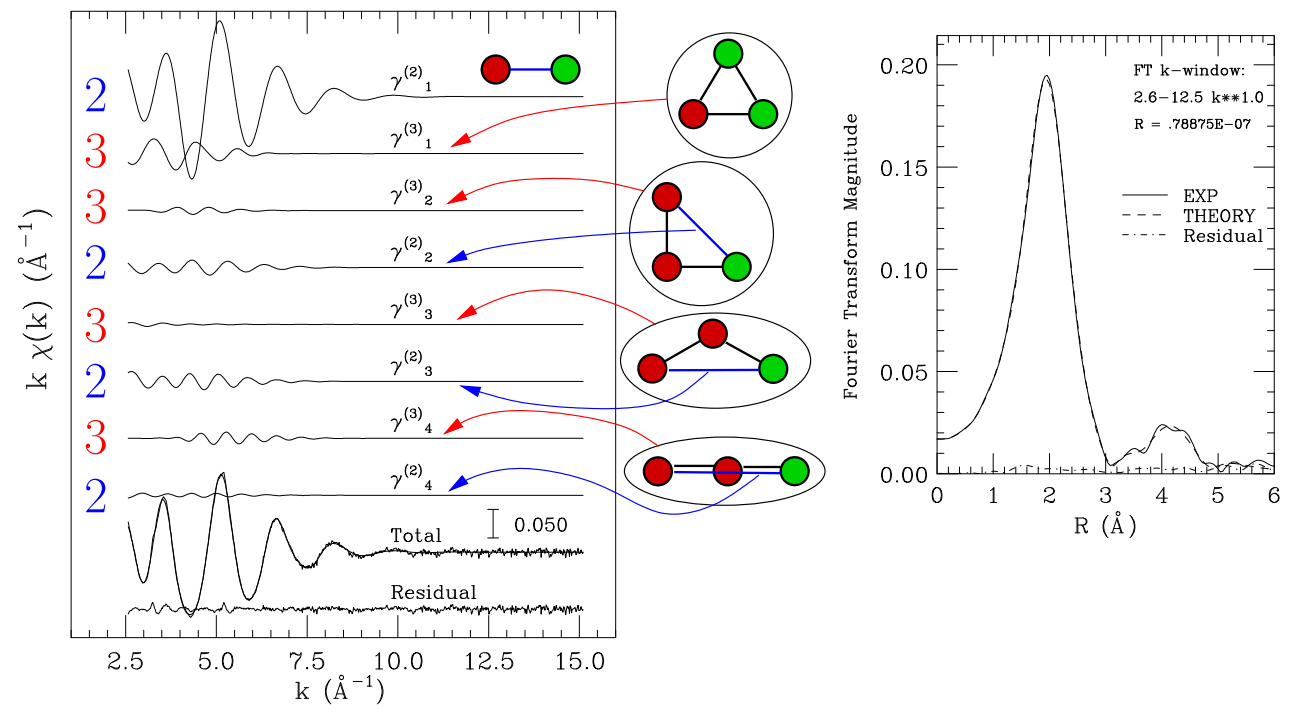

Figure 1. XAFS data analysis of the f.c.c. solid Ni at $T=1493 \mathrm{~K}$, the various pair $\gamma^{(2)}$ or triplet $\gamma^{(3)}$ oscillating contributions to the $k \chi(k)$ spectrum associated with the first shell or triangular atomic configurations involving first neighbors are reported in the left panel. The Fourier transform magnitude of the experimental, theoretical, and residual signal are shown in the right hand side panel.

and triplet contributions required to model the spectrum up to $R \approx 5 \AA$ and the related Fourier transformations are reported in figure 1 . The relevant triangular structures are indicated in the graphical insets: the inequivalent possible positions of the photoabsorber atoms are reported as red circles. The inter-atomic distances are schematically indicated as black or blue segments, the latter representing the successive coordination shells in the f.c.c. structure with associated $\gamma_{1,2,3,4}^{(2)}$ signals. The dominant first shell signal $\gamma_{1}^{(2)}$ is due to the 12 first neighbors. The distance distribution was modeled with a slightly asymmetric Gamma distribution with skewness $\beta=0.33(5)$, average distance $R=2.485(5) \AA$ and variance $\sigma^{2}=0.033(4) \AA^{2}$ compatible with the phonon contribution at this temperature. The other signals associated with triangular configuration are displayed separately for the pair $\gamma_{2,3,4}^{(2)}$ or irreducible triplet $\gamma_{1,2,3,4}^{(3)}$ contributions. The relatively strong triplet signal from equilateral triangles can be appreciated. In the case of $90^{\circ}$ and especially $120^{\circ}$ triangles, the triplet signal is much weaker than 
the corresponding pair signal from the (second or third) shell, the opposite occurs for aligned atomic configurations (fourth shell) due to the well known focusing effect. The vibrational damping of all these signals can be calculated assuming Gaussian three-dimensional distributions of the geometrical parameters defining the triangles. Their inclusion can be done avoiding a proliferation of fitting parameters since they involve first neighbor bonds whose $R$ and $\sigma^{2}$ parameters are already constrained by the $\gamma_{1}^{(2)}$ signal and only the angular fluctuations (of the order of $1^{\circ}$ ) and vibrational correlations have to be included. The fitted correlations are negligible apart form the one between the vibrations of adjacent aligned bonds that results $\rho_{R, R^{\prime}}=-0.19(2)$.

The fit is excellent and the residual difference signal (experiment-model) is substantially flat and dominated by random noise (or residual weak higher frequency neglected contributions). The possibility to model accurately the spectra of a known Ni structure provides a strong evidence for the validity of the underlying approximations and the possibility of applications to cases in which the structure is unknown.

\section{A peak fitting analysis of the spectrum of undercooled liquid $\mathrm{Ni}$}

The analysis of XAS spectra of liquid structures requires a proper account for the configurational average and limitations in the application of a peak fitting approach were clearly stated [8]. The peak fitting approach, however, can be adopted as a preliminary and effective tool to investigate the information content in a signal, and this analysis is presented here for the case of undercooled liquid $\mathrm{Ni}$ at $T_{u}=1493 \mathrm{~K}$ [14]. The experimental spectrum is slightly weaker and shifted with respect to the one of the f.c.c. crystal at the same $T$.

A central issue in this kind of data analysis is that the signal should be fitted with structural contributions compatible with a disordered three-dimensional close-packed structure in which the atomic environments may differ considerably from the one in the f.c.c. crystal and include distorted or defected icosahedral configurations, resulting in an angular distribution between nearest neighbor bonds similar to the one shown in Fig. 3 of Ref. [14]. Multiple scattering calculations however show that for all intermediate angles (similarly to the triplet signals for the $90^{\circ}$ and $120^{\circ}$ triangles in the f.c.c. structure) the triplet contributions are negligible, so a detectable signal may only arise from the limit $60^{\circ}$ (and possibly $180^{\circ}$ ) configurations that are present in any close-packed disordered configuration. The question addressed here is if the experimental signal can be modeled by accounting only for the pair signals associated with the radial distribution function, or if any signal from triplet configuration survives and can be detected. The answer is given by the fit reported in figure 2 .

The first signal (on the left hand side panel from the top) accounts for the dominant shortrange part of the radial distribution, compatible with the previous analysis [14]. This signal however in not able to account completely for the experimental data since it leaves out a higher frequency oscillation that is out of phase with respect to an hypothetical pair signal with the same frequency. By attempting to include the dominant triplet configurations associated with equilateral triangles or $180^{\circ}$ alignments, similarly to the fit of the f.c.c. Ni spectrum of figure 1 , an excellent fit can be obtained. The $180^{\circ}$ alignments (the reported $\eta_{2}^{(3)}$ signal includes both pair and triplet contributions) give only a weak signal due to the increased disorder in the liquid phase, and the present analysis cannot be regarded as conclusive. On the other hand a clear signal survives from the triplet equilateral configurations involving first neighbors. This signal has a reduced intensity with respect to the f.c.c. one, as a consequence of the increased bond distance variance $\sigma^{2}=0.044(5) \AA^{2}$ of the first $g(r)$ peak with respect to the f.c.c. case. In addition its intensity was further reduced by about $80 \%$ indicating a reduction of the number or equilateral triangles/atom in the disordered structure. This analysis 

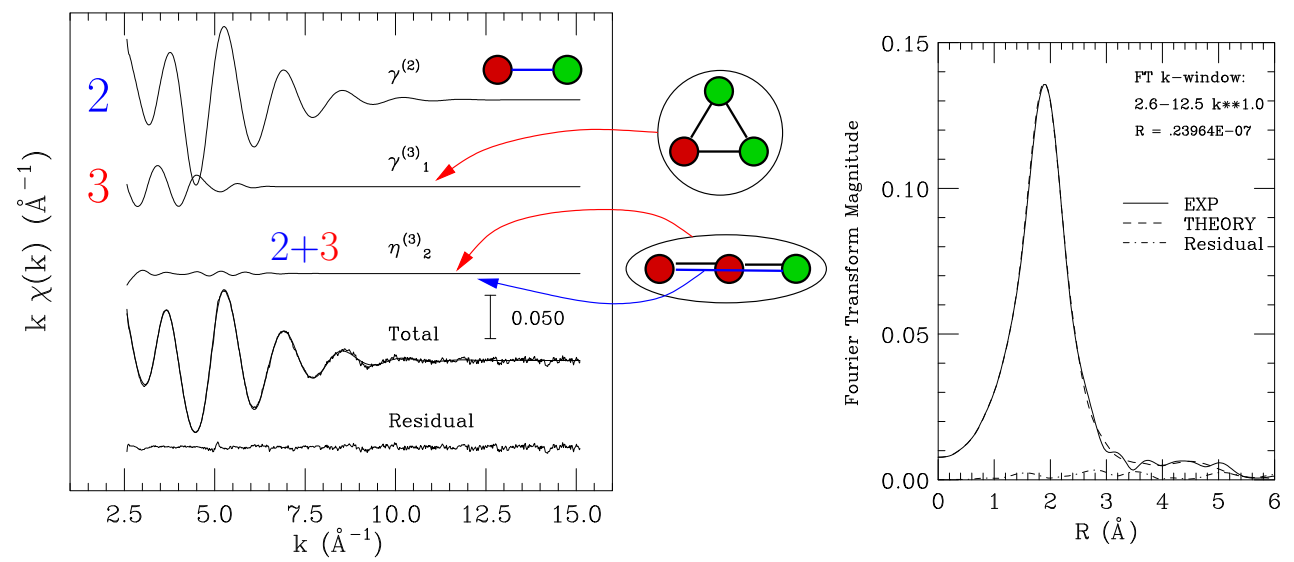

Figure 2. XAFS data analysis of the spectrum of undercooled liquid $\mathrm{Ni}$ at $T_{u}=1493 \mathrm{~K}$ accounting for all short-range pair contributions $\gamma^{(2)}$, equilateral triangular configurations $\gamma_{1}^{(3)}$, and configurations at $180^{\circ} \eta_{2}^{(3)}$ (including both pair and triplet). The Fourier transform magnitude of the experimental, theoretical, and residual signals are shown in the right hand side panel.

was performed taking account of the dominant peak of the triplet distribution modeled with an uncorrelated Gaussian shape. While this model is clearly inadequate to account for the exact entire triangle distribution in a disordered liquid structure, the presence of this signal provides a clear signature of the close packed nature of the liquid structure.

\section{Conclusions}

X-ray absorption spectroscopy provides a unique probe for the structure of elemental or multi-component liquids with accessible x-ray absorption edges. The structural information content is complementary to the one provided by x-ray or neutron diffraction experiments because of the greater sensitivity to the short-range characteristics of the radial distribution function that can be reliably constrained by a XAFS spectrum. Moreover, and especially in the case of close packed liquid metal structures, a clear signature from triplet correlations associated with close packed equilateral triangular structures can be detected, as this work demonstrates for the $\mathrm{Ni}$ case. These structures are at the basis of the polytetrahedral atomic arrangements [24] expected in the case of close packed elemental liquids and the access to experimental information on triplet correlations is clearly a unique and valuable characteristic of XAS. Present evidence shows that, for the Ni case, the signal accounts for about the $10-20 \%$ of the total signal and is confined to the low- $k$ range $k \lesssim 5 \AA^{-1}$.

Our current data analysis approach based on the Reverse Monte Carlo simulation of a three dimensional model structure compatible with the experimental spectrum was so far mostly confined to $k \geq 4 \AA^{-1}[13,14]$. The contribution from three body configurations detectable in the experimental data was accounted for by subtracting from the experimental data the three body signal for a Gaussian three-dimensional distribution of equilateral triangles formed by first neighbours, whose compatibility with the simulated average structure was verified as a self-consistency check. The structure models were anyway analyzed to extract information of the bond-angle distributions between first-neighbor bonds and using structural indicators of the local symmetry ( $W_{6}$ invariants or common neighbor analysis) that 
revealed the presence of an increased icosahedral short-range order upon undercooling. Ongoing efforts are devoted to implement the direct inclusion of the triplet signal in the RMC optimization procedure and to the extension of the fitting $k$ range down to $k \simeq 2.5 \AA^{-1}$ where the theoretical modeling was shown to be still accurate. It is believed that these computational efforts will better constrain the resulting three-dimensional structures and provide a deeper insight into their microscopic details.

\section{References}

[1] C. Notthoff, H. Franz, M. Hanfland, D.M. Herlach, D. Holland-Moritz, W. Petry, Rev. Sci. Instrum. 71, 3791 (2000)

[2] L. Hennet, I. Pozdnyakova, A. Bytchkov, V. Cristiglio, P. Palleau, H.E. Fischer, G.J. Cuello, M. Johnson, P. Melin, D. Zanghi et al., Rev. Sci. Instrum. 77, 053903 (2006)

[3] T. Schenk, D. Holland-Moritz, V. Simonet, R. Bellissent, D.M. Herlach, Phys. Rev. Lett. 89, 075507 (2002)

[4] K.F. Kelton, G.W. Lee, A.K. Gangopadhyay, R.W. Hyers, T.J. Rathz, J.R. Rogers, M.B. Robinson, D.S. Robinson, Phys. Rev. Lett. 90, 195504 (2003)

[5] G.W. Lee, A.K. Gangopadhyay, K.F. Kelton, R.W. Hyers, T.J. Rathz, J.R. Rogers, D.S. Robinson, Phys. Rev. Lett. 93, 037802 (2004)

[6] J.J. Rehr, R.C. Albers, Rev. Mod. Phys. 72, 621 (2000)

[7] J.A. Van Bokhoven, C. Lamberti, eds., X-Ray Absorption and X-Ray Emission Spectroscopy: Theory and Applications (Wiley, New York, 2016), ISBN 978-1-118-84423-6

[8] A. Filipponi, J. Phys.: Condens. Matter 13, R23 (2001)

[9] A. Filipponi, P. D'Angelo, in X-Ray Absorption and X-Ray Emission Spectroscopy: Theory and Applications, edited by J.A. Van Bokhoven, C. Lamberti (Wiley, New York, 2016), chap. 25, pp. 745-771

[10] L. Ottaviano, A. Filipponi, A. Di Cicco, Phys. Rev. B 49, 11749 (1994)

[11] A. Filipponi, A. Di Cicco, S. De Panfilis, Phys. Rev. Lett. 83, 560 (1999)

[12] A. Di Cicco, G. Aquilanti, M. Minicucci, A. Filipponi, J. Rybicki, J. Phys.: Condens. Matter 11, L43 (1999)

[13] A. Di Cicco, A. Trapananti, S. Faggioni, A. Filipponi, Phys. Rev. Lett. 91, 135505 (2003)

[14] A. Di Cicco, F. Iesari, S. De Panfilis, M. Celino, S. Giusepponi, A. Filipponi, Phys. Rev. B 89, 060102(R) (2014)

[15] A. Di Cicco, A. Trapananti, E. Principi, S. De Panfilis, A. Filipponi, Appl. Phys. Lett. 89, 221912 (2006)

[16] R. Poloni, S. De Panfilis, A. Di Cicco, G. Pratesi, E. Principi, A. Trapananti, A. Filipponi, Phys. Rev. B 71, 184111 (2005)

[17] A. Filipponi, M. Borowski, P.W. Loeffen, S. De Panfilis, A. Di Cicco, F. Sperandini, M. Minicucci, M. Giorgetti, J. Phys.: Condens. Matter 10, 235 (1998)

[18] S. De Panfilis, A. Filipponi, J. Appl. Phys. 88, 562 (2000)

[19] A. Filipponi, A. Di Cicco, S. De Panfilis, P. Giammatteo, F. Iesari, Acta Mater. 124, 261 (2017)

[20] A. Filipponi, P. Giammatteo, J. Chem. Phys. 145, 211913 (2016)

[21] A. Filipponi, A. Di Cicco, C.R. Natoli, Phys. Rev. B 52, 15122 (1995)

[22] A. Filipponi, A. Di Cicco, Phys. Rev. B 52, 15135 (1995)

[23] A. Filipponi, P. D’Angelo, J. Chem. Phys. 109, 5356 (1998)

[24] F. Spaepen, Nature 408, 781 (2000) 